\title{
Emergence of Clostridium difficile tcdC variant 078 in Marseille, France
}

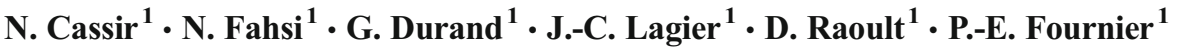

Received: 7 March 2017 / Accepted: 22 May 2017 /Published online: 1 June 2017

(C) Springer-Verlag Berlin Heidelberg 2017

\begin{abstract}
The purpose of this investigation was to evaluate the epidemiology of hypervirulent Clostridium difficile ribotypes from January 2013 to February 2017 in the Marseille area of southern France. By using the Xpert Clostridium difficile Epi polymerase chain reaction (PCR) assay and sequencing the $t c d C$ gene, we characterised $C$. difficile isolates from symptomatic patients diagnosed with $C$. difficile infection (CDI) in Marseille university hospitals. We first tested retrospectively 278 C. difficile samples isolated from January 2013 to December 2014 and observed a high prevalence of isolates with $t c d C$ mutations and deletions previously described in both hypervirulent ribotypes RT027 and RT078 (16.4\% and 10.7\%, respectively). We highlighted the co-circulation of these two hypervirulent $C$. difficile tcd $C$ variants (tV) with distinct epidemiological characteristics. While an RT027 outbreak occurred mainly as healthcare-associated infection in the elderly, CDI caused by tV078 occurred mainly in a younger population as community-associated infection. From January 2016, a systematic survey of these two hypervirulent $C$. difficile ribotypes revealed the emergence of CDI caused by tV078, currently being more prevalent than RT027 in the Marseille area. The present study is the first report of the emergence of CDI caused by tV078 in southern France. We showed the simultaneous circulation and sequential spread of hypervirulent ribotypes,
\end{abstract}

N. Cassir

cassirnadim@gmail.com

P.-E. Fournier

pierre-edouard.fournier@univ-amu.fr

1 Unité de Recherche sur les Maladies Infectieuses Tropicales et Emergentes (URMITE), Aix Marseille Université, UM63, CNRS 7278, IRD 198, INSERM 1095, Institut Hospitalier Universitaire (IHU)-Méditerranée Infection, 19-21 Boulevard Jean Moulin, 13385 Marseillle Cedex 05, France such as RT027 and tV078. This emphasises the need for an efficient surveillance system for CDI with ribotyping and an optimised management of CDI caused by hypervirulent strains.

\section{Introduction}

Clostridium difficile is the leading cause of healthcareassociated diarrhoea in developed countries, representing a great clinical and economical burden [1]. This infection usually occurs in elderly patients with co-morbidities in whom the gut microbiota has been disrupted by a previous antibiotic therapy [2]. Over the past decade, the hypervirulent fluoroquinolone-resistant polymerase chain reaction (PCR) ribotype NAP1/027 C. difficile (RT027), associated with an increased severity and mortality, emerged worldwide [3]. From January 2013 to October 2014, an RT027 outbreak occurred in the Marseille area, southeastern France [4, 5]. In total, 19 different healthcare facilities reported 112 RT027 infections, with a mortality rate of $31.2 \%$. Successful interventions included the monitoring of the regional RT027 burden and the cohorting of RT027-positive patients in a specialised infectious diseases ward. In addition, an optimised management including faecal microbiota transplantation (FMT) led to a significant reduction of mortality [6].

However, other hypervirulent $C$. difficile ribotypes (RT), including RT017, RT078 and RT244, have also emerged recently $[3,7]$, and some studies reported an increase in community-associated cases caused by these RTs, affecting younger patients $[8,9]$. In France, data from the National Reference Laboratory for $C$. difficile indicate that toxinotype V/ribotype 078 significantly increased $(3.25 \%$ vs. $11.1 \%)$ in northern France from 2006 to 2007 (comparison period: July to December) [10]. In the present study, we retrospectively sequenced the $t c d C$ gene for genotyping $278 C$. difficile 
strains collected in our laboratory during the RT027 outbreak that developed from January 2013 to December 2014 in the Marseille area. The aim was to retrospectively evaluate whether more than one hypervirulent ribotype circulated during this outbreak. In a second aim, we prospectively and specifically searched in 2016 the most common hypervirulent RTs (i.e. RT027 and RT078) in all patients diagnosed with $C$. difficile infection (CDI) in Marseille university hospitals.

\section{Materials and methods}

\section{Definitions}

Patients were considered to have CDI if they had diarrhoea ( $\geq 3$ unformed stools per $24 \mathrm{~h}$ ) and a stool sample positive for C. difficile toxin by laboratory assay. Severe CDI was defined if at least one of the following signs was present in the absence of another explanation: white blood count $(\mathrm{WBC})>15 \times 10^{9} /$ $\mathrm{L}$; fever (core body temperature $>38.5^{\circ} \mathrm{C}$ ); colectomy; ileus; megacolon; peritonitis; septic shock requiring intensive care unit (ICU) admission; serum creatinine concentration $>50 \%$ above the baseline; serum albumin concentration $<30 \mathrm{~g} / \mathrm{L}$; death. Community-associated CDI was defined as a case that either had a diagnosis of CDI in the outpatient setting with no history of hospital discharge in the 12 weeks before diagnosis, or a primary diagnosis upon hospital admission and no history of hospital discharge in the 12 weeks before diagnosis. Patients not fulfilling these criteria were diagnosed as having healthcare-associated CDI. An outbreak in a healthcare facility was defined as the occurrence of $\geq 2$ epidemiologically linked cases within one week. Mortality was considered to be attributable to CDI when a patient died of the consequences of CDI during hospitalisation.

\section{Detection and genotyping of $C$. difficile isolates}

Of the 614 stool specimens from patients with CDI diagnosed from January 2013 to December 2014 using the Xpert C. difficile Epi PCR assay (Cepheid, la Serre, France) in the two point-of-care routine laboratories located in the Timone and North university hospitals in Marseille [11], we retrospectively studied the 278 (45.2\%) samples for which at least $20 \mu \mathrm{L}$ of DNA remained. The Xpert $C$. difficile Epi assay detects the genes encoding toxin $\mathrm{B}(t c d B)$ and the binary toxin $(c d t)$, as well as the $t c d C$ gene deletion at nt117, allowing to detect PCR ribotype 027 C. difficile strains [12]. We also amplified by PCR and sequenced a fragment of the $t c d C$ gene coding for a protein regulating the $C$. difficile toxin secretion, as previously described [13]. This fragment has the advantage of being variable according to the ribotype. For example, in addition to the above-described 18-base-pair deletion at position 117, RT027 has a mutation at the same position, while the $t c d C$ variant $\mathrm{tV} 078$ has a 39-base-pair deletion and a mutation at position 184 (C instead of T).

In a second aim, from January to January 2017 (inclusive), all stool specimens positive for $C$. difficile in Marseille public hospitals were systematically tested using the same method in order to differentiate RT027 and tV078 from other $C$. difficile ribotypes.

\section{Statistical analysis}

PASW Statistics version 17.0 was used for the statistical analysis. Mean \pm standard deviation was used to describe continuous variables. Percentages and numbers of events were used for quantitative variables. Student's $t$-test or the Mann-Whitney test, when appropriate, were used to perform two-group comparisons for quantitative variables. The Chi-squared test or Fisher's exact test, when appropriate, were used for qualitative variables. A significance threshold of 0.05 was adopted for all of the statistical analyses.

\section{Results}

We first tested retrospectively 278 C. difficile samples isolated from symptomatic patients with CDI from January 2013 to December 2014 and observed a high prevalence of isolates with $t c d C$ mutations and deletions previously described in both hypervirulent ribotypes RT027 and RT078 (16.4\% and 10.7\%, respectively). We highlighted the co-circulation of these two hypervirulent C. difficile $t c d C$ variants $(\mathrm{tV})$ with distinct epidemiological characteristics. Indeed, the in-hospital mortality rates of infections caused by tV078 and RT027 during the study period were $23 \%(7 / 30)$ and $39 \%$ (18/46), respectively $(p=0.16)$. Compared with patients with RT027, patients with CDI due to tV078 were younger (69.8 years \pm 18.1 vs. 82.3 years $\pm 12.3 ; p<0.001)$ and more frequently had community-associated CDI ( $9 / 30$ vs. 3/46; $p=0.03)$. Using the European Centre for Disease Prevention and Control (ECDC) criteria, 58\% of tV078 patients and $70 \%$ of RT027 patients met the definition of severe disease $(17 / 30$ vs. $32 / 46 ; p=0.26)$. Patients with RT027 CDI were more likely to receive vancomycin than metronidazole, in line with treatment recommendations favouring oral vancomycin for severe CDI [14]. Among the 30 patients with tV078 CDI, 15 were treated with vancomycin and 11 with metronidazole, three were initially started on metronidazole and then switched to vancomycin, and one received FMT. Two cases required ICU admission.

Compared with patients with CDI due to ribotypes other than tV078 and RT027, patients with CDI due to tV078 had a 
Fig. 1 Evolution of the number of samples positive for Clostridium difficile ribotype (RT) $027, t c d C$ variant (tV) 078 and other RTs from January 2016 to February 2017 in Marseille, France

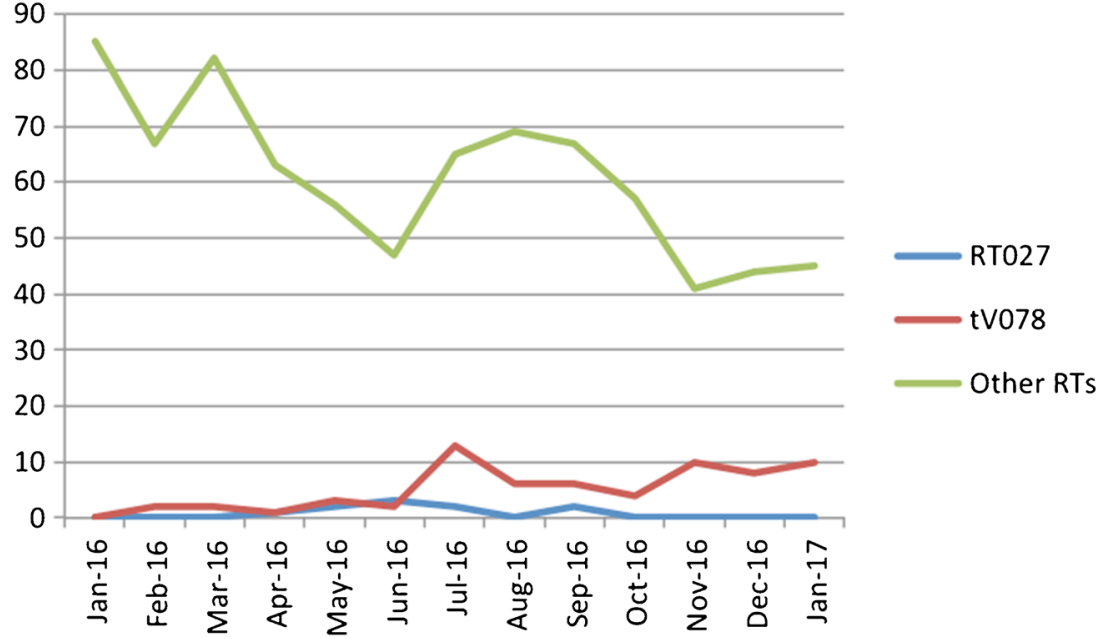

higher in-hospital mortality rate $[23 \%$ (7/30) vs. $15 \%(30 /$ $202) ; p=0.47$ ], were younger ( 69.8 years \pm 18.1 vs. 73.2 years $\pm 10.4 ; p<0.001)$ and more frequently had communityassociated CDI (9/30 vs. 20/202; $p=0.02$ ).

From January 2016 to February 2017, among all positive C. difficile strains detected in the laboratory, we identified 10 (1.2\%) RT027, 67 (7.7\%) tV078 and 788 (91.1\%) other ribotypes (Fig. 1). During the second half of 2016 (from July to December), we observed a significant increase in the rate of tV078 when compared to all $C$. difficile-positive samples [39/342 (11.4\%) vs. 10/416 (2.4\%); $p<0.0001]$. In 2016, no healthcare-associated outbreaks caused by this hypervirulent ribotype have been notified in the hospitals of the Marseille area.

\section{Discussion}

In the present study, we observed that both hypervirulent ribotypes RT027 and tV078 co-circulated from January 2013 to December 2014 in the Marseille area.

Although CDI has been considered a hospital-acquired disease mainly transmitted by symptomatic patients, recent studies have indicated the existence of other sources of $C$. difficile, showing that some CDI cases cannot be linked to a previous case of CDI $[15,16]$. Indeed, $C$. difficile is ubiquitous in the environment, and most RTs are shared among humans, animals and the environment [17]. In particular, RT078, a recently described genotype that was demonstrated to be associated with an increased morbidity and mortality and to occur in younger patients than RT027, has been detected in farm animals, especially pigs, with human and animal strains being genetically related [18]. In this study, we showed a cocirculation of two hypervirulent RTs with distinct epidemiological characteristics. While an RT027 outbreak occurred mainly as healthcare-associated infection in elderly patients, the most probable source being a single long-term care facility
[5], CDI caused by tV078 occurred mainly in a younger population as community-associated infection.

On the basis of these preliminary findings, we performed, from January 2016, a systematic survey of $C$. difficile tV078 and RT027, which revealed the emergence of CDI caused by the former ribotype, currently representing the most prevalent encountered hypervirulent ribotype in the Marseille area. Access to $C$. difficile ribotyping is crucial to detect and control CDI outbreaks according to the strains' ribotype specificities [19], hypervirulent CD ribotypes requiring optimised management and rigorous infection control measures. As we have developed specific control measures for RT027-infected patients, including cohorting in a specialised infectious diseases ward and systematic FMT, resulting in a significantly decreased mortality [6], the identification of other cocirculating hypervirulent $C$. difficile clones requiring a similar management highlights the need to prospectively and systematically detect these clones.

We acknowledge that this study has some limitations. First, the number of samples tested is low and only stools for which sufficient DNA quantity remained for genotyping procedures were analysed. Second, detailed clinical characteristics and antimicrobial susceptibility testing were not assessed. Third, the methodology used in this study allowed us to distinguish only the $C$. difficile isolates with $t c d C$ deletions and mutations previously found in ribotypes RT027 and RT078. Moreover, as mentioned before, other hypervirulent ribotypes such as RT017 or RT244 have recently been described. We intend, in a further study, to analyse, by multi-locus sequence typing (MLST), all of our C. difficile strains in order to better distinguish all the known PCR ribotypes.

\section{Conclusion}

This is the first report of the emergence of Clostridium difficile infection (CDI) caused by the $t c d C$ variant tV078 in 
southeastern France. The emergence of so-called hypervirulent $C$. difficile types has intensified the challenge of CDI epidemiology. The present study provides a comprehensive view of the current paradigm of CDI, revealing the simultaneous circulation and sequential spread of hypervirulent ribotypes. This emphasises the need for an efficient surveillance system for CDI with ribotyping and an optimised management of CDI caused by hypervirulent strains.

Acknowledgements The authors acknowledge the members of the molecular biosciences team and the staff of the infectious diseases ward at Nord hospital, Marseille, France.

\section{Compliance with ethical standards}

Financial support No financial support was provided relevant to this article.

Conflict of interest All authors report no conflicts of interest relevant to this article.

Ethical approval The institutional board approved the study protocol.

Informed consent Informed consent was not obtained from the patients, as the study design was retrospective and did not modify standard care.

\section{References}

1. Wiegand PN, Nathwani D, Wilcox MH, Stephens J, Shelbaya A, Haider S (2012) Clinical and economic burden of Clostridium difficile infection in Europe: a systematic review of healthcarefacility-acquired infection. J Hosp Infect 81:1-14. doi:10.1016/j. jhin.2012.02.004

2. Loo VG, Bourgault A-M, Poirier L, Lamothe F, Michaud S, Turgeon N et al (2011) Host and pathogen factors for Clostridium difficile infection and colonization. N Engl J Med 365:1693-1703. doi:10.1056/NEJMoa1012413

3. Freeman J, Bauer MP, Baines SD, Corver J, Fawley WN, Goorhuis B et al (2010) The changing epidemiology of Clostridium difficile infections. Clin Microbiol Rev 23:529-549. doi:10.1128/CMR. 00082-09

4. Lagier J-C, Dubourg G, Cassir N, Fournier P-E, Colson P, Richet H et al (2013) Clostridium difficile 027 emerging outbreak in Marseille, France. Infect Control Hosp Epidemiol 34:1339-1341. doi: $10.1086 / 673995$

5. Cassir N, Delarozière J-C, Dubourg G, Delord M, Lagier J-C, Brouqui $\mathrm{P}$ et al (2016) A regional outbreak of Clostridium difficile PCR-ribotype 027 infections in southeastern France from a single long-term care facility. Infect Control Hosp Epidemiol 37:13371341. doi:10.1017/ice.2016.164

6. Lagier J-C, Delord M, Million M, Parola P, Stein A, Brouqui P et al (2015) Dramatic reduction in Clostridium difficile ribotype 027associated mortality with early fecal transplantation by the nasogastric route: a preliminary report. Eur J Clin Microbiol Infect 34:1597-1601. doi:10.1007/s10096-015-2394-X

7. Lim SK, Stuart RL, Mackin KE, Carter GP, Kotsanas D, Francis MJ et al (2014) Emergence of a ribotype 244 strain of Clostridium difficile associated with severe disease and related to the epidemic ribotype 027 strain. Clin Infect Dis 58:1723-1730. doi:10.1093/cid/ ciu203

8. Goorhuis A, Bakker D, Corver J, Debast SB, Harmanus C, Notermans DW et al (2008) Emergence of Clostridium difficile infection due to a new hypervirulent strain, polymerase chain reaction ribotype 078. Clin Infect Dis 47:1162-1170. doi:10.1086/ 592257

9. Eyre DW, Tracey L, Elliott B, Slimings C, Huntington PG, Stuart RL et al (2015) Emergence and spread of predominantly community-onset Clostridium difficile PCR ribotype 244 infection in Australia, 2010 to 2012. Euro Surveill 20:21059

10. Rupnik M, Widmer A, Zimmermann O, Eckert C, Barbut F (2008) Clostridium difficile toxinotype V, ribotype 078, in animals and humans. J Clin Microbiol 46:2146. doi:10.1128/JCM.00598-08

11. Cohen-Bacrie S, Ninove L, Nougairède A, Charrel R, Richet H, Minodier P et al (2011) Revolutionizing clinical microbiology laboratory organization in hospitals with in situ point-of-care. PLoS One 6:e22403. doi:10.1371/journal.pone.0022403

12. Babady NE, Stiles J, Ruggiero P, Khosa P, Huang D, Shuptar S et al (2010) Evaluation of the Cepheid Xpert Clostridium difficile Epi assay for diagnosis of Clostridium difficile infection and typing of the NAP1 strain at a cancer hospital. J Clin Microbiol 48:45194524. doi:10.1128/JCM.01648-10

13. Baldan R, Trovato A, Bianchini V, Biancardi A, Cichero P, Mazzotti $\mathrm{M}$ et al (2015) Clostridium difficile PCR ribotype 018, a successful epidemic genotype. J Clin Microbiol 53:2575-2580. doi:10.1128/JCM.00533-15

14. Debast SB, Bauer MP, Kuijper EJ; European Society of Clinical Microbiology and Infectious Diseases (2014) European Society of Clinical Microbiology and Infectious Diseases: update of the treatment guidance document for Clostridium difficile infection. Clin Microbiol Infect 20(Suppl 2):1-26. doi:10.1111/1469-0691.12418

15. Eyre DW, Cule ML, Wilson DJ, Griffiths D, Vaughan A, O'Connor $\mathrm{L}$ et al (2013) Diverse sources of $C$. difficile infection identified on whole-genome sequencing. N Engl J Med 369:1195-1205. doi:10. 1056/NEJMoa1216064

16. Walker AS, Eyre DW, Wyllie DH, Dingle KE, Harding RM, O'Connor L et al (2012) Characterisation of Clostridium difficile hospital ward-based transmission using extensive epidemiological data and molecular typing. PLoS Med 9:e1001172. doi: 10.1371/ journal.pmed.1001172. http://www.ncbi.nlm.nih.gov/pmc/articles/ PMC 3274560

17. Janezic S, Zidaric V, Pardon B, Indra A, Kokotovic B, Blanco JL et al (2014) International Clostridium difficile animal strain collection and large diversity of animal associated strains. BMC Microbiol 14:173. doi:10.1186/1471-2180-14-173

18. Knetsch CW, Connor TR, Mutreja A, van Dorp SM, Sanders IM, Browne HP et al (2014) Whole genome sequencing reveals potential spread of Clostridium difficile between humans and farm animals in the Netherlands, 2002 to 2011. Euro Surveill 19:20954

19. Barbut F, Mastrantonio P, Delmée M, Brazier J, Kuijper E, Poxton I et al (2007) Prospective study of Clostridium difficile infections in Europe with phenotypic and genotypic characterisation of the isolates. Clin Microbiol Infect 13:1048-1057. doi:10.1111/j.14690691.2007.01824.x 